\title{
Maximum mass of hyperon stars with the Nijmegen ESC08 model
}

\author{
H.-J. Schulze \\ INFN Sezione di Catania, Dipartimento di Fisica, Via Santa Sofia 64, I-95123 Catania, Italy \\ T. Rijken \\ IMAPP, University of Nijmegen, 6525 AJ Nijmegen, The Netherlands \\ (Received 21 June 2011; published 6 September 2011)
}

\begin{abstract}
We perform Brueckner-Hartree-Fock calculations of hypernuclear matter employing the recent Nijmegen ESC08 hyperon-nucleon potentials, provide useful parametrizations of the numerical results, and compute the structure of hyperon (neutron) stars within this approach. Very low maximum masses below 1.4 solar masses are found.
\end{abstract}

DOI: 10.1103/PhysRevC.84.035801

PACS number(s): 26.60.Kp, 13.75.Ev

\section{INTRODUCTION}

The recent claim of discovery of a two-solar-mass neutron star (NS) [1] reinforces the importance of theoretical calculations of neutron star structure and their interpretation in confrontation with observational data. It has long been known that the appearance of "exotic" components (quarks, mesons, hyperons) in $\beta$-stable NS matter might strongly soften the equation of state (EOS) and reduce the theoretical maximum mass of the star [2]. Confronting accurate theoretical predictions with observational data, one might therefore be able to draw conclusions regarding the presence of these particles in stellar matter, and thus on the features of the underlying fundamental interactions.

This is in particular the case for hyperons, which are expected to appear in $\beta$-stable matter already at relatively low densities of about twice nuclear saturation density. If this is the case, the existence of heavy NS's would rule out the presence of hyperons in their interior, and require alternative scenarios. It is therefore of great importance to carry out accurate theoretical calculations of hypernuclear matter and the corresponding hyperon star structure, as much as possible constrained by independent experimental information on the hyperon-nucleon interactions.

This article is located in this framework, namely, it studies the possible appearance of hyperons in NS matter within the Brueckner-Hartree-Fock (BHF) theoretical manybody approach, continuing several earlier publications [3-7]. The fundamental input of these parameter-free calculations are the realistic potentials in the nucleon-nucleon $(\mathrm{NN})$, hyperon-nucleon (YN), and hyperon-hyperon (YY) sectors, supplemented by three-body forces (TBF's), which at least in the $\mathrm{NN}$ sector are required in order to ensure a correct saturation point of nuclear matter.

Currently several high-quality NN potentials are available for theoretical calculation, together with nuclear TBF's which have been either determined empirically by fitting the saturation point of nuclear matter $[8,9]$, or constructed in a microscopic way, compatible with the two-body potential that is used [10-12]. There exist also several YN potentials fitted to scattering data, while the potentials in the YY sector and TBF's involving hyperons have presently to be considered rather uncertain or unknown, which is basically due to the lack of appropriate experimental data and/or the great difficulties of their theoretical analysis.

We have in the past performed BHF calculations of hypernuclear matter using the Argonne $V_{18} \mathrm{NN}$ potential [13], together with the phenomenological Urbana UIX' $\mathrm{TBF}^{1}[8,9]$ and the Nijmegen NSC89 [16] or NSC97 [17] YN potentials. Very low maximum masses of hyperon stars, below $1.4 M_{\odot}$, were always found [5,6].

The purpose of this work is to extend those calculations by employing recently developed $\mathrm{YN}$ potentials and nuclear TBF's. More precisely, we will present results obtained with the Nijmegen ESC08b YN potentials [18] and the microscopic nuclear TBF developed in Ref. [12]. Both changes are expected to stiffen the resulting EOS and could thus possibly allow larger maximum masses of hyperon stars than found before, for the following reasons: The ESC08b YN potential features in particular a repulsive $\Sigma^{-} \mathrm{N}$ interaction in agreement with recent experimental indications [19], and in contrast to the previously used YN potentials. Therefore a smaller $\Sigma^{-}$ fraction is now expected in $\beta$-stable matter. Furthermore, the microscopic TBF used now is more repulsive than the previous UIX' TBF, leading to much larger masses of nucleonic stars $[6,9]$.

We have, however, stressed in previous works the existence of an important self-regulating compensation mechanism that always leads to rather low neutron star masses by the appearance of hyperons; namely, a stiffer nucleonic EOS will cause an earlier onset of hyperons and thus a stronger softening effect, and vice versa. The same is true for individual components of the hyperon EOS, e.g., a more repulsive $\Sigma$-nucleon interaction is expected to lead to an earlier onset of the $\Lambda$, etc. It is thus of particular interest to see this mechanism at work with the new calculation presented here.

\footnotetext{
${ }^{1}$ The prime denotes the fact that within the BHF approach the two parameters $A$ and $U$ of the TBF are different from the original values in [14] in order to fit the saturation point of symmetric nuclear matter. The result is a less repulsive EOS, leading to smaller maximum masses than in the variational calculation of [15].
} 


\section{FORMALISM}

We first provide a short review of the BHF approach including hyperons (detailed accounts can be found in Refs. [3] and [4]) and then describe briefly the new features of the Nijmegen ESC08 potentials.

\section{A. BHF approach to neutron star structure}

The basic input quantities in the Bethe-Goldstone equation are the $\mathrm{NN}, \mathrm{YN}$, and YY potentials. In this work we use the Argonne $V_{18}$ NN potential [13] supplemented by the microscopic TBF of Ref. [12], and the recent Nijmegen extended soft-core ESC08b YN potentials [18], that are well adapted to the existing experimental YN scattering data.

With these potentials, the various $G$ matrices are evaluated by solving numerically the Bethe-Goldstone equation, which can be written in operatorial form as

$$
G_{a b}[W]=V_{a b}+\sum_{c} \sum_{p, p^{\prime}} V_{a c}\left|p p^{\prime}\right\rangle \frac{Q_{c}}{W-E_{c}+i \varepsilon}\left\langle p p^{\prime}\right| G_{c b}[W],
$$

where the indices $a, b, c$ indicate pairs of baryons and the Pauli operator $Q_{c}$ and energy $E_{c}$ characterize the propagation of intermediate baryon pairs. The pair energy in a given channel $c=\left(B_{1} B_{2}\right)$ is

$$
E_{\left(B_{1} B_{2}\right)}=T_{B_{1}}\left(k_{B_{1}}\right)+T_{B_{2}}\left(k_{B_{2}}\right)+U_{B_{1}}\left(k_{B_{1}}\right)+U_{B_{2}}\left(k_{B_{2}}\right)
$$

with $T_{B}(k)=m_{B}+k^{2} / 2 m_{B}$, where the various single-particle potentials are given by

$$
U_{B}(k)=\sum_{B^{\prime}=n, p, \Lambda, \Sigma^{-}} U_{B}^{\left(B^{\prime}\right)}(k)
$$

and are determined self-consistently from the $G$ matrices,

$U_{B}^{\left(B^{\prime}\right)}(k)=\sum_{k^{\prime}<k_{F}^{\left(B^{\prime}\right)}} \operatorname{Re}\left\langle k k^{\prime}\left|G_{\left(B B^{\prime}\right)\left(B B^{\prime}\right)}\left[E_{\left(B B^{\prime}\right)}\left(k, k^{\prime}\right)\right]\right| k k^{\prime}\right\rangle$.

The coupled Eqs. (1) to (4) define the BHF scheme with the continuous choice of the single-particle energies. It has been shown that with this choice the nuclear EOS can be calculated with good accuracy in the Brueckner two-hole-line approximation, and that the results in this scheme are quite close to the calculations which also include the three-hole-line contribution [20]. In contrast to the standard purely nucleonic calculation, the additional coupled-channel structure due to hyperons renders the calculations quite time-consuming.

Once the different single-particle potentials are known, the total nonrelativistic baryonic energy density $\varepsilon$ can be evaluated:

$$
\varepsilon=\sum_{B=n, p, \Lambda, \Sigma^{-}} \sum_{k<k_{F}^{(B)}}\left[T_{B}(k)+\frac{1}{2} U_{B}(k)\right]=\varepsilon_{N}+\varepsilon_{Y},
$$

where

$$
\begin{aligned}
\varepsilon_{N} & =\sum_{N, N^{\prime}=n, p} \sum_{\substack{k<k_{F}^{(N)} \\
\varepsilon_{Y}}}\left[T_{N}(k)+\frac{1}{2} U_{N}^{\left(N^{\prime}\right)}(k)\right], \\
& \sum_{\substack{Y, Y^{\prime}=\Lambda, \Sigma^{-} \\
N=n, p}} \sum_{\substack{k<k_{F}^{(Y)} \\
N=n}}\left[T_{Y}(k)+U_{Y}^{(N)}(k)+\frac{1}{2} U_{Y}^{\left(Y^{\prime}\right)}(k)\right] .
\end{aligned}
$$

Knowing the baryonic energy density Eq. (5), and adding the contributions of the noninteracting leptons, the various chemical potentials $\mu_{i}=\partial \varepsilon / \partial \rho_{i}$ (of the species $i=$ $\left.n, p, \Lambda, \Sigma^{-}, e, \mu\right)$ can be computed straightforwardly and the equations for $\beta$ equilibrium, $\mu_{i}=b_{i} \mu_{n}-q_{i} \mu_{e}$ ( $b_{i}$ and $q_{i}$ denoting baryon number and charge of species $i$ ), and charge neutrality, $\Sigma_{i} \rho_{i} q_{i}=0$, allow one to determine the equilibrium composition $\left\{\rho_{i}(\rho)\right\}$ at given baryon density $\rho$ and finally the EOS:

$$
p(\rho)=\rho^{2} \frac{d}{d \rho} \frac{\varepsilon\left(\left\{\rho_{i}(\rho)\right\}\right)}{\rho}=\rho \frac{d \varepsilon}{d \rho}-\varepsilon .
$$

Knowing the EOS, the equilibrium configurations of static neutron stars are obtained by solving the TolmanOppenheimer-Volkoff (TOV) equations [2] for the pressure $p(r)$ and the enclosed mass $m(r)$,

$$
\begin{aligned}
\frac{d p}{d r} & =-\frac{G m \varepsilon}{r^{2}} \frac{(1+p / \varepsilon)\left(1+4 \pi r^{3} p / m\right)}{1-2 G m / r}, \\
\frac{d m}{d r} & =4 \pi r^{2} \varepsilon
\end{aligned}
$$

being $G$ the gravitational constant. Starting with a central mass density $\varepsilon(r=0) \equiv \varepsilon_{c}$, one integrates out until the surface density equals that of iron. This gives the stellar radius $R$ and its gravitational mass $M=m(R)$. For the description of the NS crust, we join the hadronic EOS with those by Negele and Vautherin [21] in the medium-density regime, and those by Feynman-Metropolis-Teller [22] and Baym-PethickSutherland [23] for the outer crust.

\section{B. The Nijmegen ESC08 potential}

The extended soft-core ESC08 model [18] for baryonbaryon interactions of the SU(3) flavor octet of baryons ( $N, \Lambda, \Sigma$, and $\Xi$ ) provides a presentation of the forces in terms of (i) meson exchange, using generalized soft-core Yukawa functions; (ii) multiple gluon exchange (pomeron and odderon); and (iii) structural effects due to the quark core of the baryons. Relativistic effects are included via expansions in inverse baryon masses $1 / m_{B}$. The ESC meson-exchange interactions contain local and nonlocal potentials due to (a) one boson exchanges (OBE's), which are members of nonets of pseudoscalar, vector, scalar, and axial mesons; (b) pomeron and odderon exchanges; (c) two pseudoscalar exchanges (TME's); and (d) meson pair exchanges (MPE's). The OBE and MPE vertices are regulated by Gaussian form factors, where the assignment of the cutoff masses for the baryon-baryon-meson (BBM) vertices depends on the SU(3) classification of the exchanged mesons for OBE, and a similar scheme for MPE. 
TABLE I. Fit parameters for the energy density of hypernuclear matter, Eqs. (11) through (15), obtained with the ESC08 or the NSC89 YN potentials.

\begin{tabular}{|c|c|c|c|c|c|c|c|c|c|c|c|c|c|c|}
\hline \multirow{4}{*}{$\begin{array}{l}a_{0}, b_{0}, c_{0}, a_{1}, b_{1}, c_{1} \\
a_{\Lambda}^{0}, a_{\Lambda}^{1}, a_{\Lambda}^{2}, b_{\Lambda}^{0}, b_{\Lambda}^{1}, b_{\Lambda}^{2}, c_{\Lambda} \\
a_{\Sigma}^{0}, a_{\Sigma}^{1}, a_{\Sigma}^{2}, b_{\Sigma}^{0}, b_{\Sigma}^{1}, b_{\Sigma}^{2}, c_{\Sigma}\end{array}$} & \multicolumn{7}{|c|}{$\mathrm{V} 18+\mathrm{TBF}+\mathrm{ESC} 08$} & \multicolumn{7}{|c|}{ V18 + UIX' + NSC89 } \\
\hline & -140.7 & 390.1 & 2.08 & 88.3 & 634.3 & 3.11 & & -286.6 & 397.2 & 1.39 & 88.1 & 207.7 & 2.50 & \\
\hline & -625 & 67 & 0 & 656 & -17 & 0 & 1.28 & -403 & 688 & -943 & 659 & -1273 & 1761 & 1.72 \\
\hline & -1285 & -395 & 0 & 1856 & -93 & 0 & 1.07 & -114 & 0 & 0 & 291 & 0 & 0 & 1.63 \\
\hline$a_{\Lambda \Lambda}, c_{\Lambda \Lambda}, d_{\Lambda \Lambda}$ & 218 & 0.95 & 0.84 & & & & & 136 & 0.51 & 0.93 & & & & \\
\hline$a_{\Lambda \Sigma}, c_{\Lambda \Sigma}, d_{\Lambda \Sigma}$ & 0 & 0 & 0 & & & & & 0 & 0 & 0 & & & & \\
\hline$a_{\Sigma \Lambda}, c_{\Sigma \Lambda}, d_{\Sigma \Lambda}$ & 157 & 0.95 & 0.80 & & & & & 89 & 0.33 & 0.81 & & & & \\
\hline$c_{\Lambda}^{0}, c_{\Lambda}^{1}, c_{\Sigma}^{0}, c_{\Sigma}^{1}$ & -0.13 & 1.76 & -0.75 & -0.44 & & & & 0.22 & -0.38 & -0.59 & -0.22 & & & \\
\hline
\end{tabular}

The ESC models describe the NN, YN, and YY interactions in a unified way using broken flavor $\mathrm{SU}(3)$ symmetry. This serves to connect the $\mathrm{NN}$, YN, and $\mathrm{YY}$ channels and is utilized to make a simultaneous fit to the $\mathrm{NN}$ and $\mathrm{YN}$ data
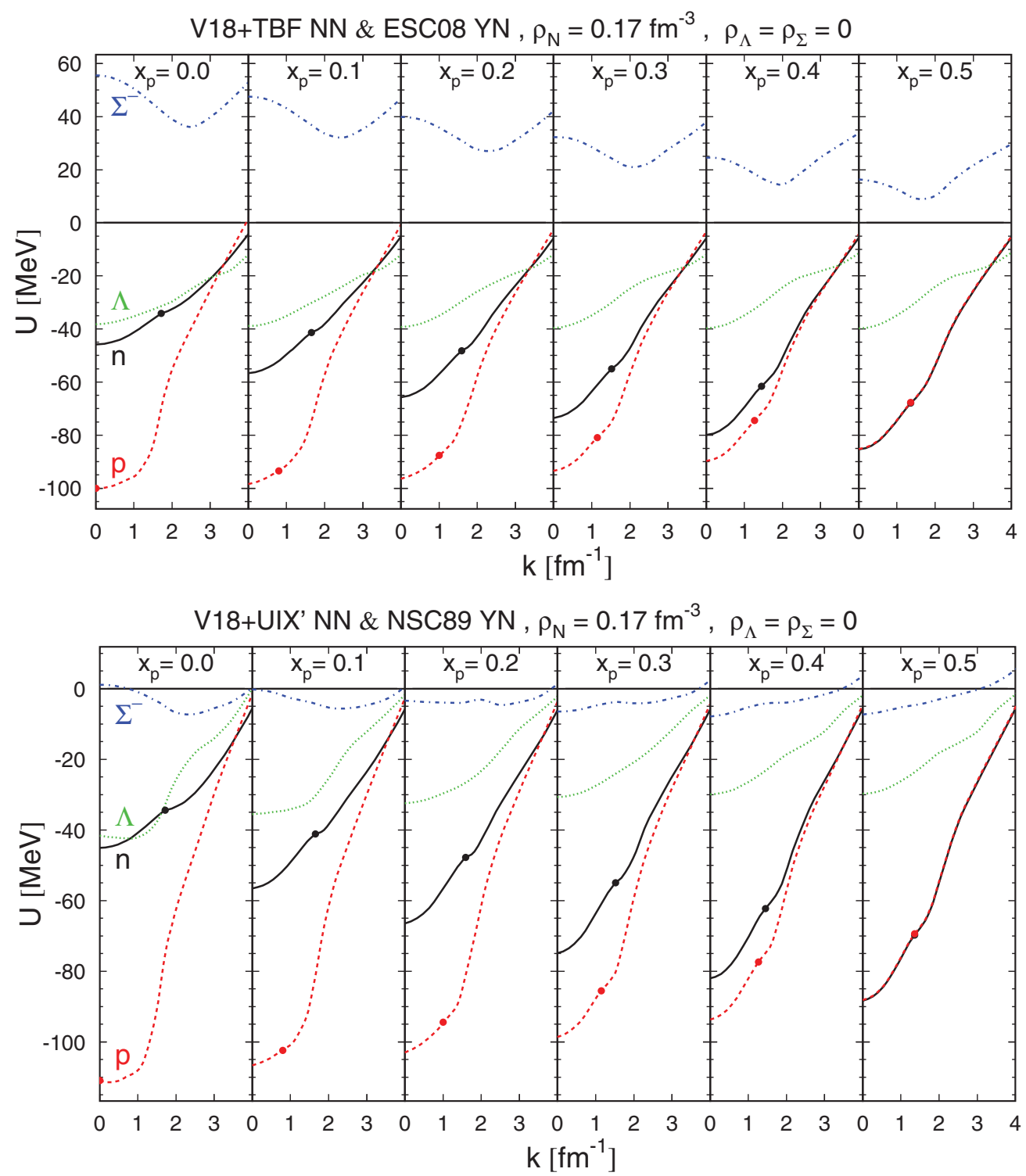

FIG. 1. (Color online) Single-particle potentials of the different species $n, p, \Lambda, \Sigma^{-}$in nuclear matter of normal nuclear density $\rho_{N}=$ $0.17 \mathrm{fm}^{-3}$, varying proton fraction $x_{p}$, and vanishing hyperon densities. The dots indicate the positions of the Fermi momenta. Results obtained with the ESC08 (upper panel) and NSC89 (lower panel) YN potentials are compared. 
with a restricted set $(\leqslant 20)$ of free coupling constants, etc.; see [18] for details. In particular, the BBM coupling constants are calculated via SU(3) using, together with the meson mixing angles, the fitted constants in the $\mathrm{NN} \oplus \mathrm{YN}$ analysis as input. In ESC08 no breaking of SU(3) is assumed for the couplings with the exception of the charge symmetry breaking in the $\Lambda p$ and $\Lambda n$ channels. In the latter we include the $\mathrm{SU}(2)$ isospin breaking in the OBE, TME, and MPE potentials.

The ESC08 model achieves, with single sets of parameters and without ad hoc changes of the rules in particular channels, excellent results for the NN and YN data: (i) For the selected $4233 \mathrm{NN}$ data of the Nijmegen phase shift analysis [24] with energies $0 \leqslant T_{\text {lab }} \leqslant 350 \mathrm{MeV}$ a $\chi^{2} /$ data $=1.094$ is realized, which is remarkably close to that of the multienergy phase shift analysis [24]. (ii) For the usual set of $38 \mathrm{YN}$ data used in the Nijmegen studies the fit gives $\chi^{2} /$ data $\simeq 0.83$. (iii) For YY there is a weak $\Lambda \Lambda$ attraction, which matches the Nagara event [25]. Among the predictions for the $S=-2$ channels $(\Lambda \Lambda, \Xi N, \Lambda \Sigma, \Sigma \Sigma)$ are the existence of $S=-2$ bound states in the $\Xi N\left({ }^{3} S_{1}-{ }^{3} D_{1}, T=0,1\right)$ channels.

\section{RESULTS}

\section{A. Parametrization of the energy density function}

The large number of degrees of freedom (four partial densities) renders inconvenient the use of the resulting hypernuclear EOS in tabular form. We therefore tried to approximate the numerical results by a sufficiently accurate analytical parametrization. We find that the following functional form provides an excellent fit of the numerical data for the energy density, Eq. (5), in the required ranges of nucleon density $\left(0.1 \mathrm{fm}^{-3} \lesssim \rho_{N} \lesssim 0.8 \mathrm{fm}^{-3}\right)$, proton fraction $(0.0 \leqslant$ $\left.\rho_{p} / \rho_{N} \leqslant 0.5\right)$, and hyperon fractions $\left(0 \leqslant \rho_{\Lambda} / \rho_{N} \leqslant 1.0,0 \leqslant\right.$ $\left.\rho_{\Sigma} / \rho_{N} \leqslant 0.5\right):$

$$
\begin{aligned}
& \varepsilon\left(\rho_{n}, \rho_{p}, \rho_{\Lambda}, \rho_{\Sigma}\right)=E_{N} \rho_{N} \\
& \quad+\left(E_{\Lambda}+E_{\Lambda \Lambda}+E_{\Lambda \Sigma}\right) \rho_{\Lambda}+\frac{C}{2 m_{\Lambda} M_{\Lambda}} \rho_{\Lambda}^{5 / 3} \\
& \quad+\left(E_{\Sigma}+E_{\Sigma \Sigma}+E_{\Sigma \Lambda}\right) \rho_{\Sigma}+\frac{C}{2 m_{\Sigma} M_{\Sigma}} \rho_{\Sigma}^{5 / 3}
\end{aligned}
$$

with

$$
\begin{aligned}
E_{N} & =(1-\beta)\left(a_{0} \rho_{N}+b_{0} \rho_{N}^{c_{0}}\right)+\beta\left(a_{1} \rho_{N}+b_{1} \rho_{N}^{c_{1}}\right), \\
E_{Y} & =\left(a_{Y}^{0}+a_{Y}^{1} x+a_{Y}^{2} x^{2}\right) \rho_{N}+\left(b_{Y}^{0}+b_{Y}^{1} x+b_{Y}^{2} x^{2}\right) \rho_{N}^{c_{Y}}, \\
E_{Y Y^{\prime}} & =a_{Y Y^{\prime}} \rho_{N}^{c_{Y Y^{\prime}}} \rho_{Y^{\prime}}^{d_{Y Y^{\prime}}} \\
M_{Y} & =1+\left(c_{Y}^{0}+c_{Y}^{1} x\right) \rho_{N},
\end{aligned}
$$

where $\rho_{N}=\rho_{n}+\rho_{p}, x=\rho_{p} / \rho_{N}, \beta=(1-2 x)^{2}, Y, Y^{\prime}=$ $\Lambda, \Sigma$, and $C=(3 / 5)\left(3 \pi^{2}\right)^{2 / 3} \approx 5.742$. Here $\varepsilon$ and $\rho_{i}$ are given in units of $\mathrm{MeV} \mathrm{fm}{ }^{-3}$ and $\mathrm{fm}^{-3}$, respectively (and $m_{\Lambda, \Sigma}$ in $\mathrm{MeV}^{-1} \mathrm{fm}^{-2}$ ).

Technically, these parametrizations were obtained by performing about $10^{3} \mathrm{BHF}$ calculations in the $\left(\rho_{n}, \rho_{p}, \rho_{\Lambda}, \rho_{\Sigma}\right)$ space, yielding "data" points $\varepsilon\left(\rho_{n}, \rho_{p}, \rho_{\Lambda}, \rho_{\Sigma}\right)$. The optimal values of the fit parameters were then determined hierarchically first for nuclear matter, and then for hypernuclear matter, so that the fits are optimized also for pure nuclear matter. The optimal parameters are listed in Table I for both the V18 + $\mathrm{TBF}+\mathrm{ESC} 08$ and the V18 + UIX' + NSC89 interactions that we compare in this article. The final overall $\mathrm{rms}$ deviation of fit and BHF data points for $E / A=\varepsilon / \rho$ is less than $1.5 \mathrm{MeV}$, which we consider fully satisfactory for our current purposes.

\section{B. Neutron star structure}

In order to illustrate the major differences between the ESC08 and NSC89 YN potentials, we compare in Fig. 1 the BHF single-particle potentials $U_{B}(k)$, Eq. (3), of the different species $B=n, p, \Lambda, \Sigma^{-}$in nuclear matter of normal nuclear density $\rho_{N}=0.17 \mathrm{fm}^{-3}$, varying proton fraction $x_{p}$, and vanishing hyperon densities. The most striking difference is the strong repulsion of the $\Sigma^{-}$obtained with the ESC08 model: $U_{\Sigma^{-}}(0)$ ranges from about $15 \mathrm{MeV}$ in symmetric matter to $55 \mathrm{MeV}$ in neutron matter, whereas with the NSC89 model its value is close to zero. The $\Lambda$ is slightly more attractive with the ESC08 in nuclear matter $(-40 \mathrm{MeV}$ vs $-30 \mathrm{MeV})$, while the values in neutron-rich matter are nearly the same. One can thus expect reduced $\Sigma^{-}$concentrations in $\beta$-stable matter with the ESC08.

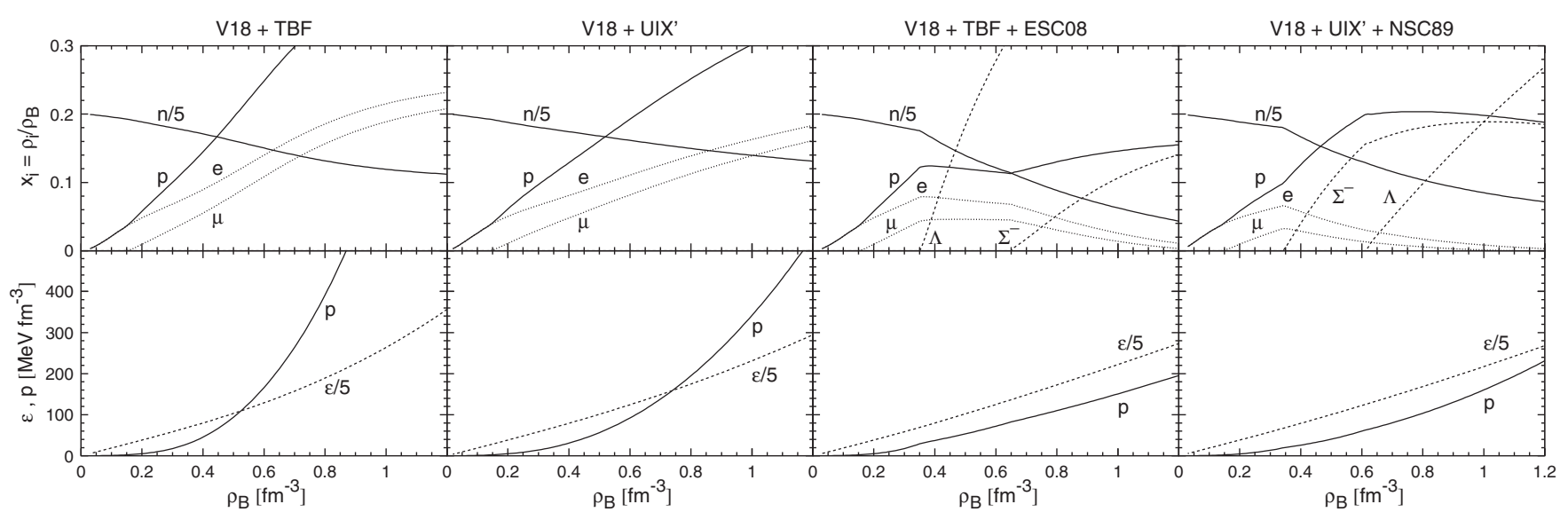

FIG. 2. Composition of $\beta$-stable matter (upper panels) and equation of state (lower panels) for different models. 


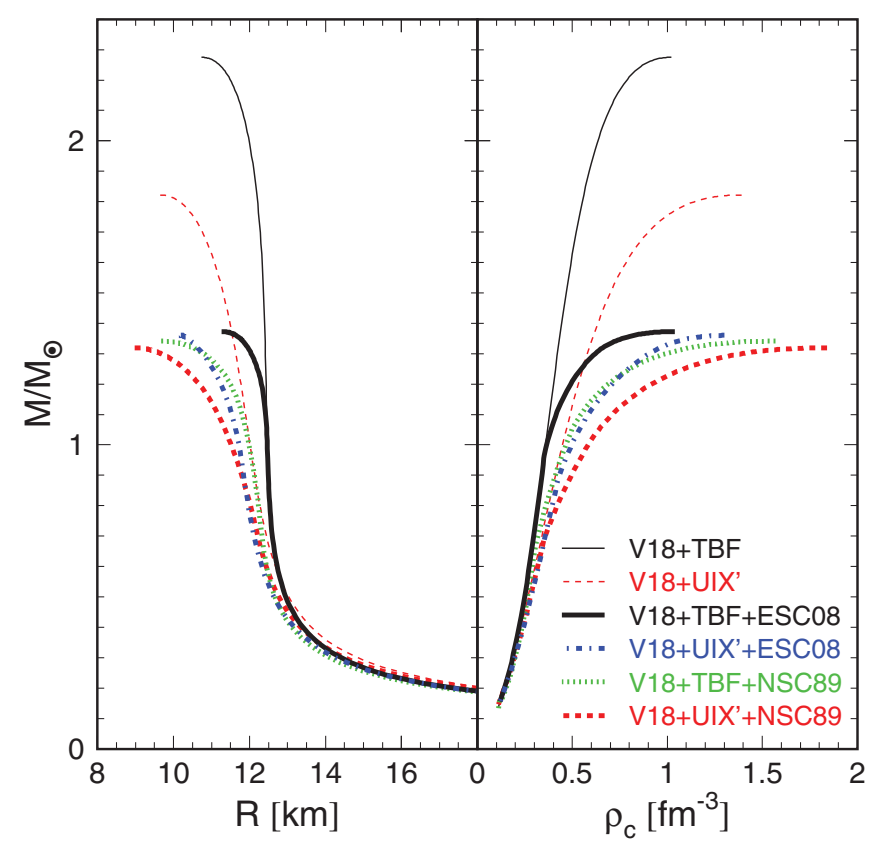

FIG. 3. (Color online) Mass—radius and mass—central density relations for different equations of state. Details are given in the text.

This is in fact confirmed by the composition of NS matter shown in the upper panels of Fig. 2, where the results obtained with the two models V18 + TBF + ESC08 and V18 + UIX' + NSC89 are compared with those of purely nuclear matter, $\mathrm{V} 18+\mathrm{TBF}$ and $\mathrm{V} 18+\mathrm{UIX}^{\prime}$, disregarding the appearance of hyperons. It is striking to see how the roles of the $\Lambda$ and $\Sigma^{-}$hyperons are reversed with the two YN potentials: With the NSC89 the $\Sigma^{-}$appears first at about twice normal nuclear matter density and the $\Lambda$ at about $0.6 \mathrm{fm}^{-3}$, whereas with the ESC08 the hyperon onset densities are nearly the same, but $\Lambda$ and $\Sigma^{-}$are swapped. Furthermore, with the ESC08 the $\Lambda$ concentration reaches much larger values than with the NSC89, while the $\Sigma^{-}$remains more suppressed, due to its strong repulsion in neutron-rich matter; see Fig. 1.

Pressure and energy density of hyperonic NS matter, shown in the lower panels of Fig. 2, are quite similar for both models. This is in contrast to the purely nucleonic calculations, where one observes a much stiffer nuclear EOS with the microscopic TBF than with the UIX', see also Refs. [6,9]. The proton fraction is larger with the microscopic TBF, which would favor also a larger $\Sigma^{-}$concentration. Evidently this effect is completely overcome by the strong $\Sigma^{-}$repulsion with the ESC08 potential.

These results allow to interpret easily the final resulting mass-radius and mass-central density relations for the different EOS that are shown in Fig. 3: Regarding the purely nucleonic cases (thin curves), in accordance with the EOS shown in Fig. 2 one obtains a much larger maximum mass with the microscopic TBF than with the UIX' $\left(2.27 M_{\odot}\right.$ vs $1.82 M_{\odot}$ ) [6], while remarkably the introduction of hyperons yields nearly the same maximum mass in both models (1.37 $M_{\odot}$ vs $1.32 M_{\odot}$; thick solid and dashed curves). These values are also very close to the result $1.34 M_{\odot}$ that was obtained in an approximate way in Ref. [6] by combining the microscopic TBF with the NSC89 potential, i.e., V18 + $\mathrm{TBF}+\mathrm{NSC} 89$, and that we repeat here for completeness, together with the result for V18 + UIX' + ESC08 $\left(1.36 M_{\odot}\right)$, obtained in the same way.

While the maximum masses of hyperon stars are thus nearly identical, there are significant differences for the corresponding radii that are linked to the maximum central baryon density that is reached in the different models. In any case, however, most current observed NS masses [26] are superior to these theoretical values of hyperon stars.

\section{CONCLUSIONS}

In this article the finding of very low maximum masses of hyperon stars within the BHF approach is reconfirmed, using very recent realistic nucleon-nucleon and hyperon-nucleon interactions.

Compared to previous results based on the V18 + UIX' NN force and the NSC89/97 YN models, both changes are in principle able to stiffen the EOS and increase the maximum mass (as clearly shown for purely nucleonic stars), but it is amazing to see how well the self-regulating compensation softening mechanism for the hypernuclear EOS works, finally yielding nearly the same maximum mass of about $1.35 M_{\odot}$ as before.

This result reinforces once more the important conclusion that in our approach massive neutron stars have to be hybrid stars containing a core of nonbaryonic ("quark") matter [27], since the possibility of them being nucleonic stars is ruled out by the early appearance of hyperons.

It seems difficult to avoid this conclusion, even in view of the current uncertainties regarding hyperon-hyperon and hyperonic three-body interactions. Only simultaneous strong repulsion in all relevant channels could significantly raise the maximum mass (see, however, Ref. [28]). Obviously it will be an important task for the future to verify this by following future experimental and theoretical developments in this field.

\section{ACKNOWLEDGMENTS}

We would like to acknowledge valuable discussions with E. Hiyama, T. Motoba, M. M. Nagels, and Y. Yamamoto.
[1] P. B. Demorest, T. Pennucci, S. M. Ransom, M. S. E. Roberts, and J. W. T. Hessels, Nature 467, 1081 (2010).

[2] S. L. Shapiro and S. A. Teukolsky, Black Holes, White Dwarfs and Neutron Stars (Wiley, New York, 1983); N. K. Glendenning,
Compact Stars: Nuclear Physics, Particle Physics and General Relativity, 2nd ed. (Springer, Berlin, 2000); H. Heiselberg and M. Hjorth-Jensen, Phys. Rep. 328, 237 (2000); J. SchaffnerBielich, J. Phys. G 31, S651 (2005); Nucl. Phys. A 804, 309 (2008). 
[3] H.-J. Schulze, M. Baldo, U. Lombardo, J. Cugnon, and A. Lejeune, Phys. Rev. C 57, 704 (1998); M. Baldo, G. F. Burgio, and H.-J. Schulze, ibid. 58, 3688 (1998); 61, 055801 (2000).

[4] I. Vidaña, A. Polls, A. Ramos, M. Hjorth-Jensen, and V. G. J. Stoks, Phys. Rev. C 61, 025802 (2000).

[5] H.-J. Schulze, A. Polls, A. Ramos, and I. Vidaña, Phys. Rev. C 73, 058801 (2006).

[6] Z. H. Li and H.-J. Schulze, Phys. Rev. C 78, 028801 (2008).

[7] G. F. Burgio and H.-J. Schulze, Astron. Astrophys. 518, A17 (2010); G. F. Burgio, H.-J. Schulze, and A. Li, Phys. Rev. C 83, 025804 (2011).

[8] M. Baldo, I. Bombaci, and G. F. Burgio, Astron. Astrophys. 328, 274 (1997); M. Baldo and L. S. Ferreira, Phys. Rev. C 59, 682 (1999).

[9] X. R. Zhou, G. F. Burgio, U. Lombardo, H.-J. Schulze, and W. Zuo, Phys. Rev. C 69, 018801 (2004).

[10] P. Grangé, A. Lejeune, M. Martzolff, and J.-F. Mathiot, Phys. Rev. C 40, 1040 (1989).

[11] W. Zuo, A. Lejeune, U. Lombardo, and J.-F. Mathiot, Nucl. Phys. A 706, 418 (2002); Eur. Phys. J. A 14, 469 (2002).

[12] Z. H. Li, U. Lombardo, H.-J. Schulze, and W. Zuo, Phys. Rev. C 77, 034316 (2008).

[13] R. B. Wiringa, V. G. J. Stoks, and R. Schiavilla, Phys. Rev. C 51, 38 (1995).

[14] B. S. Pudliner, V. R. Pandharipande, J. Carlson, S. C. Pieper, and R. B. Wiringa, Phys. Rev. C 56, 1720 (1997).

[15] A. Akmal, V. R. Pandharipande, and D. G. Ravenhall, Phys. Rev. C 58, 1804 (1998).

[16] P. M. M. Maessen, Th. A. Rijken, and J. J. de Swart, Phys. Rev. C 40, 2226 (1989).

[17] V. G. J. Stoks and Th. A. Rijken, Phys. Rev. C 59, 3009 (1999).
[18] T. Rijken, M. Nagels, and Y. Yamamoto, Nucl. Phys. A 835, 160 (2010); Y. Yamamoto, E. Hiyama, and T. Rijken, ibid. 835, 350 (2010); T. Rijken, M. Nagels, and Y. Yamamoto, Prog. Theor. Phys. Suppl. 185, 14 (2010); Y. Yamamoto, T. Motoba, and T. Rijken, ibid. 185, 72 (2010).

[19] H. Noumi et al., Phys. Rev. Lett. 89, 072301 (2002); P. K. Saha et al., Phys. Rev. C 70, 044613 (2004); M. Kohno, Y. Fujiwara, Y. Watanabe, K. Ogata, and M. Kawai, ibid. 74, 064613 (2006).

[20] H. Q. Song, M. Baldo, G. Giansiracusa, and U. Lombardo, Phys. Rev. Lett. 81, 1584 (1998); M. Baldo, G. Giansiracusa, U. Lombardo, and H. Q. Song, Phys. Lett. B 473, 1 (2000); M. Baldo, A. Fiasconaro, H. Q. Song, G. Giansiracusa, and U. Lombardo, Phys. Rev. C 65, 017303 (2001).

[21] J. W. Negele and D. Vautherin, Nucl. Phys. A 207, 298 (1973).

[22] R. P. Feynman, N. Metropolis, and E. Teller, Phys. Rev. 75, 1561 (1949).

[23] G. Baym, C. Pethick, and P. Sutherland, Astrophys. J. 170, 299 (1971).

[24] V. G. J. Stoks, R. A. M. Klomp, M. C. M. Rentmeester, and J. J. de Swart, Phys. Rev. C 48, 792 (1993).

[25] H. Takahashi et al., Phys. Rev. Lett. 87, 212502 (2001).

[26] J. M. Lattimer and M. Prakash, Phys. Rep. 442, 109 (2007).

[27] G. F. Burgio, M. Baldo, P. K. Sahu, and H.-J. Schulze, Phys. Rev. C 66, 025802 (2002); M. Baldo, M. Buballa, G. F. Burgio, F. Neumann, M. Oertel, and H.-J. Schulze, Phys. Lett. B 562, 153 (2003); C. Maieron, M. Baldo, G. F. Burgio, and H.-J. Schulze, Phys. Rev. D 70, 043010 (2004); M. Baldo, G. F. Burgio, P. Castorina, S. Plumari, and D. Zappalà, Phys. Rev. C 75, 035804 (2007); T. Maruyama, S. Chiba, H.-J. Schulze, and T. Tatsumi, Phys. Rev. D 76, 123015 (2007); A. Kurkela, P. Romatschke, and A. Vuorinen, ibid. 81, 105021 (2010).

[28] I. Vidaña, D. Logoteta, C. Providência, A. Polls, and I. Bombaci, Europhys. Lett. 94, 11002 (2011). 